was analysed histologically and by ${ }^{3}[\mathrm{H}]$ thymidine incorporation. RNA expression levels were measured by QPCR.

Results: When we crossed miR-146a ${ }^{-/}$into hTNFtg mice, histological examination revealed a significant increase in synovial inflammation and even more striking a more than twofold increase in local bone destruction, due to increased generation of osteoclasts in the tarsal joints of miR-146a $\mathrm{a}^{-1} / \mathrm{hTNFtg}$ mice compared to hTNFtg mice. Interestingly, systemic bone loss was comparable in hTNFtg compared to miR-146a ${ }^{-1 /} / \mathrm{hTNF}$ tg mice, suggesting an important local role of miR-146a. Indeed, we detected increased levels of IL-1 $\beta$, TRAF6, a major target of miR-146a and RANKL, in addition the expression level of OPG was decreased locally in the paws of miR-146- $\mathrm{a}^{-1 /} / \mathrm{hTNFtg}$ compared to hTNFtg mice. By performing bone marrow transplants we could indeed show a pivotal role for miR-146a in mesenchymal cells in controlling local osteoclast generation and bone destruction. Analysis of important mesenchymal cells in arthritis, the synovial fibroblasts exhibited enhanced proliferation if miR-146a is missing, in vitro and in vivo. Moreover stimulation of these cells with IL-1 $\beta$, a prominent cytokine in arthritis which was also shown to be negatively regulated by miR-146a, led to increased expression of RANKL and TRAF6 in miR-146a deficient synovial fibroblasts.

Conclusions: These data demonstrate an important mitigating role of the miR$146 a$ in inflammatory arthritis, most importantly in local bone destruction, by controlling mesenchymal expression of osteoclastogenic factors. This shows an important anti-inflammatory role of miR-146a, which might possibly be exploited for therapeutic purposes.

Disclosure of Interest: None declared

DOI: 10.1136/annrheumdis-2017-eular.3384

\section{FRI0025 THE TAM RECEPTORS AXL AND MER PLAY A PROTECTIVE ROLE IN A TEMPORAL AND SPATIAL MANNER IN INFLAMMATORY ARTHRITIS}

C.E.J. Waterborg ${ }^{1}$, P.G. Través ${ }^{2}$, S. Beermann ${ }^{1}$, M.I. Koenders ${ }^{1}$, P.L.E.M. van Lent ${ }^{1}$, F. den Hoogen ${ }^{1}$, P.M. van der Kraan ${ }^{1}$, F.A.J. van de Loo ${ }^{1}$. ${ }^{1}$ Experimental Rheumatology, Radboudumc, Nijmegen, Netherlands; ${ }^{2}$ Molecular Neurobiology Laboratory, The Salk Institute for Biological Studies, la Jolla, CA, United States

Background: Rheumatoid arthritis (RA) is a chronic autoimmune disease characterized by an inflammatory response in synovial joints. One family of tyrosine kinase receptors that mediates a natural anti-inflammatory feedback mechanism are Tyro3, Axl and Mer (gene Mertk; TAM). The two principal TAM receptor protein ligands are Growth Arrest-Specific 6 (Gas6) and Protein $S$ (Pros1). Targeting the TAM receptors by adenoviral overexpression of their ligands alleviates experimental arthritis pathology. Notably, Gas6 is a ligand for all three receptors but with the highest affinity for AxI. In contrast, Pros1 can only activate Tyro3 and Mer but not Axl. The role of the separate TAM receptors in RA has not been elucidated.

Objectives: To reveal the individual role of the TAM receptors Axl and Mer in an experimental inflammatory model of RA.

Methods: The KRN serum transfer model of arthritis was induced by two

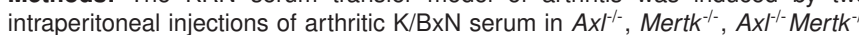
and wild-type (WT) mice. Ankle joints were macroscopically scored for 7 days. At day 0,2 and 7, ankle and knee joints were isolated for histology and immunohistochemistry.

Results: The course of arthritis was studied macroscopically and Mertk ${ }^{-1}$ mice had increased macroscopic ankle scores until day 4, compared to WT. Thereafter, $A x^{-1-}$ mice developed more severe arthritis as compared to WT with enhanced macroscopic scores from day 4 until day 7 . Histology of ankle joints showed significantly more inflammation in Mertk'- mice at day 2 and increased arthritis pathology in $\mathrm{Ax}^{-1-}$ mice at day 7 , reflecting the macroscopic ankle scores. Histological analysis of ankle joints of $A x F^{-1-}$ Mertk $^{-1-}$ mice at day 7 showed enhanced pathology compared to both $A x^{H^{-1}}$ and WT mice, indicating an additive effect of Axl and Mer deficiency. In contrast to the ankle joints at day 7, enhanced macroscopic scores and arthritis pathology in knee joints of Mertk ${ }^{-1}$ mice, compared to WT mice, was observed at both day 2 and day 7 . Axt- mice had comparable knee joint pathology compared to WT. To explain the discrepancy of Axl involvement between ankle and knee at day 7 , we examined Axl expression in synovium before the onset of arthritis. The cells in the lining layer of ankle synovium were strikingly Axl positive whereas the synovium of the knee joints was Axl negative.

Conclusions: The Mer receptor plays a protective role at the onset of arthritis whereas the Axl receptor takes over this role in established disease in ankle joints. In the knee joints, however, Mer but not Axl, plays a prominent protective role, likely due to the lack of $A x l$ in naïve knee joints. These findings identify the TAM receptors Axl and Mer as protective in arthritis, dependent on the distinct anatomic location. It is tempting to speculate that these differences in topographically distinct synovial joints could, at least in part, explain the differential joint involvement in RA.

Disclosure of Interest: None declared

DOI: 10.1136/annrheumdis-2017-eular.4887

\section{FRI0026 IDENTIFICATION OF MICRORNAS CANDIDATES SPECIFICALLY EXPRESSED IN MONOCYTES OF UNDIFFERENTIATED ARTHRITIS PATIENTS WHO PROGRESSED TO RHEUMATOID ARTHRITIS}

W. Kurowska ${ }^{1}$, E. Kuca-Warnawin ${ }^{1}$, A. Radzikowska ${ }^{1}$, M. Ciechomska ${ }^{1}$, U. Skalska ${ }^{1}$, M. Jakubaszek ${ }^{2}$, B. Kwiatkowska ${ }^{2}$, W. Maslinski ${ }^{1} .{ }^{1}$ Department of Pathophysiology and Immunology; ${ }^{2}$ Early Arthritis Clinic, National Institute of Geriatrics, Rheumatology and Rehabilitation, Warsaw, Poland

Background: Monocytes are central to the initiation of inflammation and bone erosion in rheumatoid arthritis (RA). Therefore, understanding of molecular pathways regulation in monocytes, especially at the early stages of RA development, is of crucial importance as it may help to predict the progression to the full-blown disease. However, mechanisms of gene regulation in monocytes, particularly the pattern of microRNA molecules (miRNAs) expression which can be involved in regulation of several cellular inflammatory pathways, in early phases of RA (i.e. at undifferentiated arthritis-UA stage) are not fully characterized.

Objectives: To investigate the pattern of miRNAs expression in monocytes that could serve as new biomarkers for RA development.

Methods: Magnetically sorted monocytes from peripheral blood of 19 patients with UA served for total RNA isolation. Total RNA from both sample and reference was labelled with $\mathrm{Hy} 3$ and $\mathrm{Hy} 5$ fluorescent label, respectively, using the miRCURY LNA microRNA Hi-Power Labeling Kit (Exiqon, Denmark). Fluorescent labelled samples and a reference RNA sample were hybridized to the miRCURY LNA microRNA Array 7th Gen (Exiqon), followed by scanning by Microarray Scanner System (Agilent Technologies, USA). The image analysis was carried out using ImaGene 9 Software (Exiqon).

Results: Out of 19 patients with UA enrolled in the study, 12 were verified for diagnosis after 4 years of follow-up ( 7 patients did not respond to the call for re-evaluation). Four patients developed full-blown RA (UA $\rightarrow$ RA patients), 6 patients remained still in UA phase (UA $\rightarrow$ UA patients), 1 patient was diagnosed as having Sjögren's Syndrome, and 1 undifferentiated connective tissue disease. Baseline characteristics of UA $\rightarrow R A$ vs UA $\rightarrow$ UA patients were as follows: age (median: 50.5, range 37-59 years vs 52.5, range 32-63 years), CRP (median: 9.0, range $1-22 \mathrm{mg} / \mathrm{l}$ vs 8.0 , range $1-21 \mathrm{mg} / \mathrm{l}$ ), ESR (median: 37.0 , range $4-47 \mathrm{~mm} / \mathrm{h}$ vs 34.0, range $15-44 \mathrm{~mm} / \mathrm{h}$ ) and swollen joins count (median: 3.0, range 1-9 vs 2.5 , range 1-4). Following computational unsupervised analysis we identified 50 miRNAs in monocytes that have the largest variation across all patients samples. From these 50 miRNAs we selected several specific miRNA candidates on the basis of significantly changed expression in monocytes of UA $\rightarrow R A$ vs $\cup A \rightarrow U A$ patients. Predicted specific miRNAs targeting inflammatory genes in monocytes of UA $\rightarrow$ RA patients are: miR-483-3p (2.7-fold increased, $p=0.009$ ), miR-378d (4.1-fold decreased, $p=0.0059$ ), miR-371b-5p (52.8-fold increased, $\mathrm{p}=0.0381$ ) miR-642b-5p (13.7-fold increased, $\mathrm{p}=0.0380$ ), and miR-25-3p (1.8-fold decreased, $p=0.0317$ ). Additional validation of selected miRNAs candidates will be further performed using QPCR analysis.

Conclusions: Our results indicate new miRNA candidates differentially expressed in peripheral blood monocytes from patients with UA who subsequently developed RA, in comparison to patients with UA who did not progress to RA after 4 years follow-up.

Acknowledgements: Supported by NCN Poland (grant 2012/06/A/NZ5/00059). Disclosure of Interest: None declared

DOI: 10.1136/annrheumdis-2017-eular.5703

\section{FRI0027 TNF EXPRESSION ON MICROPARTICLES FROM RHEUMATOID ARTHRITIS PATIENTS MEDIATES ENDOTHELIAL CELL FATE IN VITRO}

C. Barbati, M. Vomero, T. Colasanti, L. Novelli, F.R. Spinelli, F. Miranda,

F. Ceccarelli, A. Finucci, C. Perricone, R. Scrivo, F. Conti, G. Valesini,

C. Alessandri. Department of Internal Medicine and Medical Specialties, Arthritis Center, Sapienza University of Rome, Rome, Italy, roma, Italy

Background: Microparticles (MPs) are small membrane vesicles released by many cell types under physiological conditions or pathological situations. Increased levels of MPs have been reported in patients with autoimmune diseases, such as Rheumatoid Arthritis (RA) (1) which is characterized by an accelerated atherosclerosis. TNF, a key cytokine involved in the pathogenesis of RA, has been associated to RA atherosclerosis (2). Moreover, MPs could also have a role in endothelial dysfunction contributing to atherosclerosis in RA patients.

Objectives: The aim of this study was: 1) to evaluate TNF expression on RA-MPs. 2) to estimate the effects of serum RA-MPs on endothelial apoptosis and autophagy before and after in vivo and in vitro treatment with Etanercept. Methods: 15 RA patients were recruited from the Department of Rheumatology Sapienza University of Rome at baseline (TO) and after three months of therapy (T3) with Etanercept. A fasting blood sample was collected and centrifuged two times to obtain platelet-poor plasma rich in MPs. The resulting plasma was stained with Ab anti-TNF and analized by flow cytometry. In vitro effects of serum RA-MPs on endothelium were evaluated using human umbilical vein cell line EA.hy926. Cells were treated with RA-MPs purified at T0 and T3 and with RA-MPs in vitro treated with Etanercept. At the end of experiments apoptosis and autophagy were evaluated. Apoptosis was analyzed by flow cytometry using a FITC-conjugated 
annexin $\mathrm{V}$ and propidium iodide apoptosis detection kit; autophagy was analyzed by western blot for the expression level of the autophagic marker LC3-II. Results: Our results showed that MPs purified from RA patients at T0 expressed TNF on their surface and this expression decreased after three months of treatment with Etanercept $(p=0.04)$. Moreover, serum RA-MPs at T0 significantly increased, in a dose-dependent manner, both apoptosis and autophagy levels in the human umbilical vein cell line $E A$. hy926 $(p=0.005$ and $p=0.02$, respectively versus untreated cells). After three months of treatment with Etanercept, RA-MPs were not able to significantly change these parameters. Finally, in vitro studies showed that RA-MPs treated with Etanercept significantly decreased surface expression of TNF and were no longer able to modulate apoptosis and autophagy in EA.hy926 cells.

Conclusions: Our data demonstrate that serum RA-MPs express TNF on their surface. Moreover, both in vivo and in vitro treatment with Etanercept interfere with the ability of MPs to significantly modulate apoptosis and autophagy of endothelial cells by decreasing surface expression of TNF.

\section{References:}

[1] Beyer C and Pisetsky DS.The role of microparticles in the pathogenesis of rheumatic diseases. Nat. Rev. Rheumatol. 2010;6:21-9.

[2] Feldmann M, Brennan FM, Foxwell BM and Maini RN.The role of TNF alpha and IL-1 in rheumatoid arthritis Curr. Dir. Autoimmun. 2001;3:188-99.

Disclosure of Interest: None declared

DOI: 10.1136/annrheumdis-2017-eular.3434

\section{FRI0028 IN VITRO INHIBITORY EFFECT OF ETANERCEPT ON AUTOPHAGY: A NEW MECHANISM OF ACTION OF TNF INHIBITORS IN RHEUMATOID ARTHRITIS}

M. Vomero ${ }^{1}$, A. Capozzi ${ }^{2}$, C. Barbati ${ }^{1}$, T. Colasanti $^{1}$, V. Manganelli ${ }^{2}$, F. Ceccarelli ${ }^{1}$, F.R. Spinelli ${ }^{1}$, F. Conti ${ }^{1}$, C. Perricone ${ }^{1}$, A. Finucci ${ }^{1}$ M. Pendolino ${ }^{1}$, R. Scrivo ${ }^{1}$, R. Misasi ${ }^{2}$, M. Sorice ${ }^{2}$, G. Valesini ${ }^{1}$, C. Alessandri ${ }^{1}$ ${ }^{1}$ Department of Internal Medicine and Medical Specialties, Arthritis Center, Sapienza University of Rome; ${ }^{2}$ Department of Experimental Medicine, Sapienza University of Rome, Rome, Italy

Background: Autophagy has emerged as a key mechanism in the development, survival and function of immune cells and dysregulation of autophagic pathway has been implicated in the pathogenesis of several autoimmune diseases including Rheumatoid Arthritis (RA) (1). In fact, autophagy seems to be involved in the generation of citrullinated peptides, with consequent breakage of tolerance in RA (2). Moreover, increased autophagy levels and a reduction of apoptosis-related molecules have been found in RA synovial tissues and a role of TNF-induced autophagy in RA development has been proposed (3).

Objectives: The aim of the study was to analysed the effect of TNF and anti-TNF inhibitor etanercept on autophagy and apoptosis in cells involved in RA pathogenesis.

Methods: Peripheral blood mononuclear cells (PBMCs) and fibroblast-like synoviocytes (FLS) isolated from RA patients were cultured in presence of TNF and in serum deprivation state (starvation) for 4 hours and then etanercept, at concentration of $15 \mathrm{ug} / \mathrm{mL}$, were added to the culture. After $24 \mathrm{~h}$ cells were analyzed for levels of autophagy marker LC3-II by western blot and for percentage of annexin V-positive apoptotic cells by flow cytometry.

Results: As expected, TNF and starvation induced autophagy on RA PBMC and FLS in dose-dependent manner after $24 \mathrm{~h}$ of culture $(p<0.05$ in all experimental conditions). Moreover, the adding of etanercept caused a significant reduction of LC3-II levels $(p=0.004)$ and an increase of apoptosis rate $(p=0.002)$ after both pro-autophagic stimuli $(p<0.05)$.

Conclusions: We demonstrated for the first time an inhibitory effect of etanercept on autophagy activation of cells involved in RA pathogenesis. In addition, our findings suggest a crucial role of autophagy in RA cells survival.

\section{References:}

[1] Pierdominici M, Vomero M, Barbati C et al. Role of autophagy in immunity and autoimmunity, with a special focus on systemic lupus erythematosus. FASEB J. 2012; 26:1400-12.

[2] Sorice M, lannuccelli C, Manganelli V et al. Autophagy generates citrullinated peptides in human synoviocytes: a possible trigger for anti-citrullinated peptide antibodies. Rheumatology. 2016;55:1374-85.

[3] Rockel JS, Kapoor M. Autophagy: controlling cell fate in rheumatic diseases. Nat Rev Rheumatol. 2016;12:517-31.

Disclosure of Interest: None declared

DOI: 10.1136/annrheumdis-2017-eular.5061

\section{FRI0029 THE OXYGEN SENSOR PHD1 IS AN INDISPENSABLE REGULATOR OF ARTHRITIS DEVELOPMENT}

K. De Wilde ${ }^{1,2}$, D. Gaublomme ${ }^{1,2}$, J. Coudenys ${ }^{1,2}$, F. Windels ${ }^{1,2}$, S. Van Welden $^{3}$, A. De Muynck ${ }^{4}$, D. Elewaut ${ }^{1,2}$. ${ }^{1}$ Department of Rheumatology, Department of Internal Medicine, Ghent University; ${ }^{2}$ Unit for Molecular Immunology and Inflammation, VIB Inflammation Research Center; ${ }^{3}$ Department of Gastroenterology; ${ }^{4}$ Department of Physics and Astronomy, Ghent University, Ghent, Belgium

Background: Oxygen supply is a fundamental requirement for all living tissues.
Some tissues such as articular joints are characterized by a physiological state of hypoxia. Interestingly, under conditions of inflammation such as in arthritic disease, this level of hypoxia is even further enhanced. However, the functional significance of these observations and the molecular mechanisms involved remain poorly characterized to date. Our goal was therefore to examine the role of 3 known oxygen sensors, prolyl hydroxylase domain (PHD) proteins: PHD1, PHD2 and PHD3. They are enzymes whose function is essentially controlled by oxygen. Their expression pattern varies between either of them and all of them have been ascribed specific roles in a myriad of biological processes. [1]

Objectives: Our goal was to examine the role of oxygen sensors PHD1, PHD2 and PHD3 in preclinical models of rheumatoid arthritis, and to delineate the cellular source involved.

Methods: We subjected the collagen antibody induced arthritis (CAIA) model (resembling rheumatoid arthritis) to hypoxic $\left(10 \% \mathrm{O}_{2}\right)$ and normoxic conditions $\left(21 \% \mathrm{O}_{2}\right)$, respectively. Furthermore, the CAIA-model was induced in mice with germline deficiency of the specific PHD's and in mice with a myeloid cell-specific PHD1 deficiency versus controls. Arthritis development was assessed by clinical scoring of paw swelling, histopathology of knee joints and $\mu \mathrm{CT}$.

Results: Mice kept in hypoxic conditions during CAIA experiments showed markedly less arthritis (both by clinical and histopathological assessment) compared to mice in normoxic conditions. Furthermore, we demonstrated that PHD1 knock-out $(\mathrm{KO})$ mice had significantly less joint inflammation compared to wildtype mice. PHD1 KO mice were also protected against inflammation induced bone loss as evidenced by $\mu \mathrm{CT}$. By contrast, no differences were found between PHD2 heterozygous (PHD2 KO mice are not viable) or PHD3 KO mice and littermate controls. Because myeloid cells are considered critical effector cells upon passive transfer of arthritogenic antibodies in the CAIA model we also generated myeloid cell specific ko mice (PHD1 $\left.{ }^{\text {myelKO}}\right)$. Of interest, PHD1 $1^{\text {myelKO }}$ mice developed less arthritis compared to wildtype mice and were protected against inflammation induced bone loss.

Conclusions: Our data are consistent with a new paradigm that the oxygen sensor PHD1 is a critical regulator of myeloid cell function in arthritic disease. Overall, the data suggest that PHD1 is a potential target in the treatment of arthritis.

References:

[1] Fong G-H, Takeda K. Role and regulation of prolyl hydroxylase domain proteins. Cell Death Differ 2008;15:635-41. doi:10.1038/cdd.2008.10.

Disclosure of Interest: None declared

DOI: 10.1136/annrheumdis-2017-eular.5194

\section{FRI0030 SYNOVIAL MAST CELLS IDENTIFY PATIENTS WITH A SEVERE PHENOTYPE IN A COHORT OF DMARD NAÏVE PATIENTS WITH EARLY RHEUMATOID ARTHRITIS}

F. Rivellese ${ }^{1}$, F. Humby ${ }^{1}$, A. Nerviani ${ }^{1}$, S. Pagani ${ }^{1}$, D. Mauro ${ }^{1}$, A. de Paulis ${ }^{2}$, G. Marone ${ }^{2}$, C. Pitzalis ${ }^{1} .{ }^{1}$ Centre for Experimental Medicine \& Rheumatology, William Harvey Research Institute, Barts and the London School of Medicine \& Dentistry, London, United Kingdom; ${ }^{2}$ Department of Translational Medical Sciences (DiSMeT) and Center for Basic and Clinical Immunology Research (CISI), University of Naples Federico II, Naples, Italy

Background: Mast cells (MCs) are among the immune cells participating to the inflammatory response in Rheumatoid Arthritis (RA), but their exact contribution to disease development and progression is unclear.

Objectives: To evaluate the presence of MCs in the synovia of patients with early RA naïve to treatment and their correlation with baseline clinical phenotype, response to DMARDs and disease progression.

Methods: DMARD-naïve patients with early ( $<12$ months) RA ( $n=99)$ fulfilling the $2010 \mathrm{ACR} / \mathrm{EULAR}$ criteria were recruited as part of the Pathobiology of Early Arthritis Cohort at Barts Health NHS Trust. Sections of paraffin embedded synovial tissue obtained by ultrasound-guided synovial biopsy were stained by immunohistochemistry for CD117 (c-kit) and patients were classified into MC+ and MC- groups. Differences in clinical parameters at baseline and 6 months and progression in radiographic damage at 12 months were evaluated. The expression of Receptor Activator of Nuclear factor Kappa-B Ligand (RANKL) by human primary MCs was assessed by western blot and immunofluorescence.

Results: The presence of synovial CD117+ MCs was significantly associated with highly active disease (DAS28, ESR, CRP, tender and swollen joint counts, $p<0.05$ ). At the 6 months follow-up, there were no differences in terms of response to treatment with synthetic DMARDs (e.g. DAS28 remission $38.8 \%$ in $\mathrm{MC}+\mathrm{vs}$ $42.9 \%$ in MC-, $p=0.725)$. Nonetheless, $M C+$ patients showed a significantly higher prevalence of radiographic progression at 12 months (progressors/non progressors $13 / 38$ in $M C+$ vs $0 / 28$ in $M C-, p=0.003, n=79$ ). When progressors were compared with non progressors, there were no differences in clinical parameters at baseline (inflammatory markers, DAS28, ACPA or RF positivity). Because of the observed association of MCs with radiographic progression, we looked for mechanisms that could link MCs to bone erosions, and we found that human primary MCs express the osteoclast activator RANKL.

Conclusions: We show that synovial MCs identify patients with a severe clinical phenotype in a DMARD-naïve early RA cohort. In particular, despite a similar rate of response to DMARDs at 6 months, the presence of synovial MCs at baseline was significantly associated with radiographic progression at 12 months. As clinical parameters at baseline showed no association with radiographic 